\title{
Thermodynamic evolution theorem for chemical reactions
}

\author{
David Hochberg $\mathbb{0}^{*}$ \\ Department of Molecular Evolution, Centro de Astrobiología (CSIC-INTA), Carretera Ajalvir Kilómetro 4, \\ 28850 Torrejón de Ardoz, Madrid, Spain \\ Josep M. Ribó (1) \\ Department of Organic and Inorganic Chemistry, Institute of Cosmos Science (IEEC-UB), \\ University of Barcelona, 08028 Barcelona, Catalonia, Spain
}

(Received 2 March 2020; revised 28 April 2020; accepted 17 November 2020; published 14 December 2020)

\begin{abstract}
The production, exchange, and balance of entropy characterize the thermodynamics of open nonequilibrium systems, ranging from chemical reactions, cells, ecological systems, and Earth-like planets to stars. We generalize the Glansdorff-Prigogine general evolution criterion to constrain the entropy balance in volumetric open-flow chemical reaction systems. We derive a thermodynamic inequality governing the joint evolution of both the internal microreversible reactions and the matter fluxes that the system exchanges with its environment, as exemplified by the distribution of the entropy productions and exchanges over the chemical reaction pathways. We validate this evolution theorem and discuss the physical significance of this pathwise partitioning of the dissipation, for an autocatalytic model capable of spontaneous mirror symmetry breaking.
\end{abstract}

DOI: 10.1103/PhysRevResearch.2.043367

\section{INTRODUCTION}

Far from equilibrium systems make up for most of the applied and natural processes and the formation of structural order in energy dissipative systems [1-3]. Irreversible processes are constrained by the second law of thermodynamics and give rise to the emergence of structure and symmetry breaking in a plethora of dissipative systems in the physical and natural sciences $[4,5]$. Examples include plasma physics, where large-scale dissipative structures can augment their impedance and support high-temperature gradients while producing large quantities of entropy at smaller scales [6]. In planetary sciences, entropy production has been invoked to explain the zonal climates of Earth, Mars, and Titan [7]. Analysis of entropy production patterns can explain the diversified experimental observations and interpretations of water-rock interactions [8], as well as the spatial organization of vegetation in river basins [9]. In developmental biology, a reduction in the rate of specific entropy production has been validated in the development, growth, and aging of organisms [10]. In chemical engineering, the entropy production is employed for analyzing shock waves, phase boundaries, diffusion, convective flows, and boundary conditions [11].

A unifying thermodynamic principle for dissipative systems was established by Glansdorff and Prigogine, who derived a general inequality for the entropy production valid

\footnotetext{
*hochbergd@cab.inta-csic.es

Published by the American Physical Society under the terms of the Creative Commons Attribution 4.0 International license. Further distribution of this work must maintain attribution to the author(s) and the published article's title, journal citation, and DOI.
}

for the entire range of macroscopic physics and for fixed boundary conditions. Their result states that the temporal change of the generalized forces proceeds always in a way as to lower the value of the entropy production: This is the general evolution criterion (GEC). Although the original GEC can be applied to chemical reactions, its scope in this field has been limited to systems lacking explicit open matter flows or fluxes. Yet such flows are the hallmark of continuous open-flow systems such as, e.g., biological cells: systems that exchange matter and energy with their environments [12]. Nevertheless, the clamped approximation, which assumes constant fixed concentrations for all species external to the reaction volume, has traditionally been invoked as a convenient, if unrealistic, simplification in benefit of the mathematical analysis. The clamped approximation may well be the reason why the GEC is being largely overlooked. Hence, the extension of the GEC to volumetric open-flow systems establishes an important and needed contribution to the thermodynamic understanding of far from equilibrium nonlinear systems, and so helps broaden its range of useful applications in nonlinear dynamical systems.

In this paper, we derive a thermodynamic inequality governing the rate of change of the entropy for far from equilibrium chemical reactions driven by continuous open flow. This inequality generalizes the Glansdorff-Prigogine GEC and states that the chemical forces (the affinities $A$ ) must necessarily evolve so as to lower the temporal change in the system entropy. It provides a rigorous criterion for how the entropy balance must evolve in time. Moreover, the application of stoichiometric network analysis (SNA) $[13,14]$ reveals an equipartition of the partial entropy productions and exchanges corresponding to each individual reaction, the partitioning is over the subsets of reaction pathways in which those reactions belong. This implies that the evolution 
criterion can be expressed in terms of weighted sums of the partial entropy productions and exchanges along each reaction pathway, and summed over all the reaction pathways of the system. As a result, the chemical forces (i.e., the affinities) acting along each individual reaction pathway must evolve so as to lower the rate of change in the net system entropy $(d S / d t)$. This pathway criterion is validated, and its physical significance revealed, for an enantioselective autocatalytic model [15], which has chiral nonequilibrium stationary states that exist off the thermodynamic branch (racemic states) and is relevant in chemical evolution scenarios for explaining the prebiotic origin of biological homochirality.

\section{ENTROPY PRODUCTION AND ENTROPY EXCHANGE}

We begin with a brief synopsis of the modern approach to thermodynamics, where the changes in a system's entropy $S$ can be expressed as a balance equation [3]

$$
\frac{d S}{d t}=\frac{d_{i} S}{d t}+\frac{d_{e} S}{d t},
$$

where $d_{i} S / d t \geqslant 0$ is the non-negative rate of entropy production due to the irreversible processes within the system and $d_{e} S / d t$ is the entropy flow rate, due to the exchange of matter and energy with the exterior; see Fig. 1. The latter can be positive or negative and is zero for isolated systems. For reversible chemical reactions, the rate of entropy production per unit volume $\sigma=V^{-1} d_{i} S / d t$ in well-mixed homogeneous systems is expressed as

$$
\sigma=R \sum_{k}\left(w_{+k}-w_{-k}\right) \ln \left(\frac{w_{+k}}{w_{-k}}\right) \geqslant 0,
$$

in terms of the forward $w_{+k}$ and reverse $w_{-k}$ reaction rates of the $k$ th microreversible reaction; $R$ is the universal gas constant [3].

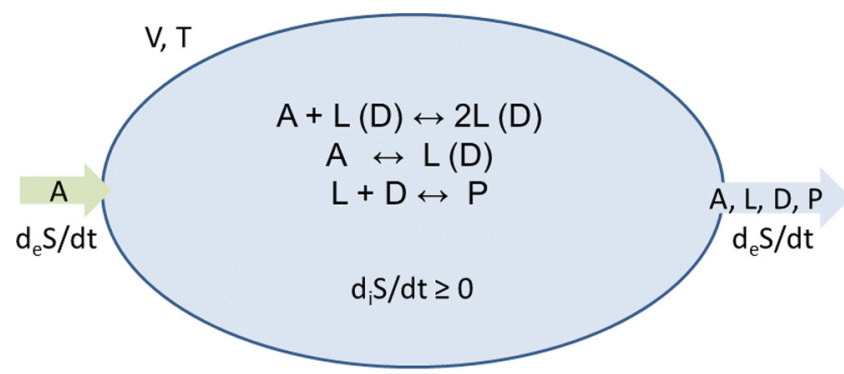

FIG. 1. Reversible version of the Frank model [15] in a continuous open-flow reactor of volume $V$ and temperature $T$, assuming instant and perfect diffusion of the all species in solution (see Appendix $C$ ). Achiral resource $A$ flows in at fixed concentration, $[A]_{\text {in }}$, and all species $A, L, D$, and $P$ flow out with their instantaneous concentrations. The matter flow maintains the system out of equilibrium. The fluid volumes entering and exiting the reactor per unit time are the same. The entropy production $d_{i} S / d t \geqslant 0$ is due to the reversible transformations taking place within $V$, the entropy exchange $d_{e} S / d t$ is due to the matter flows into and out from the reaction volume $V$.
The entropy exchange per unit volume $\sigma_{e}=V^{-1} d_{e} S / d t$ is given by [16]

$$
\sigma_{e}=R \sum_{k} f\left(x_{k}-x_{k, \text { in }}\right) \ln \left(\frac{x_{k}}{x_{k}^{\mathrm{eq}}}\right) .
$$

This depends on the concentrations of the species flowing into $\left(x_{k, i n}\right)$ and out from $\left(x_{k}\right)$ the reactor, with the volumetric flow rate $f=q / V$, where $q$ is the volume of fluid per unit time entering and exiting the reactor. The equilibrium concentrations $\left(x_{k}^{\mathrm{eq}}\right)$ are determined from detailed balance and mass conservation: the state corresponding to the reactor being isolated from the open flow $(f=q=0)$. This uses the relative chemical potential $\mu_{r e l}$ [17], which shifts the reference point of the standard potential to the equilibrium state of the system, as introduced by Onsager [18]:

$$
\begin{aligned}
\mu_{k}^{r e l} & =\left[\mu_{k}^{0}+R T \ln \left(x_{k}\right)\right]-\left[\mu_{k}^{0}+R T \ln \left(x_{k}^{\mathrm{eq}}\right)\right] \\
& =R T \ln \left(\frac{x_{k}}{x_{k}^{\mathrm{eq}}}\right) .
\end{aligned}
$$

The relative chemical potential shifts the reference point from the Gibbs energy of formation of the compound to the equilibrium state of the system (here in the case of ideal solutions where the activity is equal to the concentration). $\sigma_{e}$ is an important aspect of open-flow reactors (see Fig. 1), essential for achieving entropy balance (1) in nonequilibrium stationary states (NESS) [16]. The sum $\sigma+\sigma_{e}$ gives the entropy balance Eq. (1) per unit volume, $V^{-1} d S / d t$, and the inequality we derive below in Sec. IV shows how the evolution of this sum is related to that of the chemical affinities in the nonlinear regime of nonequilibrium thermodynamics.

\section{GEC: NICOLIS AND PRIGOGINE}

We present a brief overview of the salient points in the Nicolis and Prigogine proof of the GEC. We then highlight the key assumptions made in order to compare and contrast with our proof, valid for volumetric open-flow architectures, that follows afterward.

The entropy production $P$ associated with chemical reactions and diffusion, taking place in a system of volume $V$ and constant temperature $T$, is given by [2]

$$
\begin{aligned}
P & =\int d V \sigma=\int d V\left[-\sum_{i} j_{i} \cdot \nabla \frac{\mu_{i}}{T}+\sum_{\rho} w_{\rho} \frac{A_{\rho}}{T}\right] \\
& \equiv \int d V \sum_{k} J_{k} X_{k} .
\end{aligned}
$$

The generalized forces $X$ giving rise to the flows $J$ are those due to diffusion and chemical reactions:

$$
\begin{gathered}
X_{j}^{\text {diff }}=-\nabla \frac{\mu_{j}}{T}, \quad J_{j}^{\text {diff }}=j_{j}, \\
X_{\rho}^{\text {react }}=\frac{A_{\rho}}{T}, \quad J_{\rho}^{\text {react }}=w_{\rho},
\end{gathered}
$$

where $\mu_{j}$ is the standard chemical potential, $\boldsymbol{j}_{j}$ is the diffusive flux, $A_{\rho}$ is the affinity, and $w_{\rho}$ is the reaction rate.

Beyond the linear range of nonequilibrium thermodynamics, the total derivative $d P / d t$ does not exhibit any property of general validity, but the change in $P$ with respect to the time 
derivative of the generalized forces $X$,

$$
\begin{aligned}
\frac{d_{X} P}{d t} & =\frac{1}{T} \int d V\left[-\sum_{i} j_{i} \cdot \nabla \frac{\partial \mu_{i}}{\partial t}+\sum_{\rho} w_{\rho} \frac{\partial A_{\rho}}{\partial t}\right] \\
& \equiv \int d V \sum_{k} J_{k} \frac{\partial X_{k}}{\partial t},
\end{aligned}
$$

does satisfy a general inequality. Using Green's theorem and expressing the affinities in terms of the chemical potentials [de Donder's affinity, Eq. (20)], Nicolis and Progogine obtain

$$
\begin{aligned}
\frac{d_{X} P}{d t}= & \underbrace{-\frac{1}{T} \int d \Sigma \boldsymbol{n} \cdot \sum_{i} \boldsymbol{j}_{i} \frac{\partial \mu_{i}}{\partial t}}+\frac{1}{T} \int d V \\
& \times\left[\sum_{i j}\left(\frac{\partial \mu_{i}}{\partial \rho_{j}}\right) \frac{\partial \rho_{j}}{\partial t} \operatorname{div} \boldsymbol{j}_{i}-\sum_{i j \rho} w_{\rho} v_{i \rho}\left(\frac{\partial \mu_{i}}{\partial \rho_{j}}\right) \frac{\partial \rho_{j}}{\partial t}\right] .
\end{aligned}
$$

It is at this point of the demonstration where they assume time-independent concentrations or zero fluxes at the system's boundaries. The surface integral contribution (indicated by the underbrace) then vanishes by virtue of these boundary conditions.

Next, using the mass-balance equation [2]

$$
\frac{\partial \rho_{i}}{\partial t}=-\operatorname{div} \boldsymbol{j}_{i}+\sum_{\rho} v_{i \rho} w_{\rho}
$$

where $\rho_{i}$ is the mass density (the mass per unit volume), allows the right-hand side of Eq. (9) to be expressed as

$$
\frac{d_{X} P}{d t}=-\frac{1}{T} \int d V \sum_{i j}\left(\frac{\partial \mu_{i}}{\partial \rho_{j}}\right) \frac{\partial \rho_{i}}{\partial t} \frac{\partial \rho_{j}}{\partial t} .
$$

Lastly, in order to ensure a definite character of this quadratic form Eq. (11), Nicolis and Prigogine appeal to equilibrium thermodynamic stability conditions. For this, they use the second variation of the equilibrium thermodynamic potential (density):

$$
\left(\delta^{2} \phi_{v}\right)_{\mathrm{eq}}=\sum_{i j}\left(\frac{\partial \mu_{i}}{\partial \rho_{j}}\right)_{\mathrm{eq}} \delta \rho_{i} \delta \rho_{j} \geqslant 0,
$$

which, and in conjunction with Eq. (11), leads to the conclusion that

$$
\frac{d_{X} P}{d t} \leqslant 0 \quad(=0 \mathrm{NESS})
$$

\section{The assumptions}

There are two crucial points in the above demonstration that merit closer examination before turning to our proof below. The first point concerns the assumption of timeindependent concentrations or zero fluxes at the system's boundary. Quoting Nicolis and Prigogine [2], "these conditions correspond to an open system in communication with some external phases that are in a time-independent and spatially uniform state and are characterized by given values of temperature, pressure and chemical potentials" (p. 50). One way to implement the choice of time-independent chemical potentials for the chemical species external to the system volume is to invoke the clamped approximation. Then, a general network of elementary reactions involving $n_{a}$ external constant concentration species $A_{u}$ and $n_{x}$ reaction intermediates $X_{u}$ obeying ideal mass-action law kinetics is

$$
\sum_{u=1}^{n_{a}} \alpha_{u w}^{+} A_{u}+\sum_{u=1}^{n_{x}} \beta_{u w}^{+} X_{u} \rightleftharpoons \sum_{u=1}^{n_{a}} \alpha_{u w}^{-} A_{u}+\sum_{u=1}^{n_{x}} \beta_{u w}^{-} X_{u},
$$

where the forward and reverse extensive reaction rates are given by

$$
w_{w}^{ \pm}(\boldsymbol{a}, \boldsymbol{x})=V k_{w}^{ \pm} \prod_{u=1}^{n_{a}}\left(a_{u}\right)^{\alpha_{u w}^{ \pm}} \prod_{u=1}^{n_{x}}\left(x_{u}\right)^{\beta_{u w}^{ \pm}},
$$

and $k_{w}^{ \pm}$is the (+ forward, - reverse) rate constant, and $a_{u}, x_{u}$ are the concentrations of external and intermediate species, respectively. But since the external species $A_{u}$ have fixed concentrations, the following redefinition of the rate constants

$$
k_{w}^{\prime \pm}(\boldsymbol{a}) \equiv k_{w}^{ \pm} \prod_{u=1}^{n_{a}}\left(a_{u}\right)^{\alpha_{u w}^{ \pm}}
$$

immediately implies that the reaction rates

$$
w_{w}^{ \pm}(\boldsymbol{a}, \boldsymbol{x})=V k_{w}^{\prime \pm}(\boldsymbol{a}) \prod_{u=1}^{n_{x}}\left(x_{u}\right)^{\beta_{u w}^{ \pm}}
$$

are those corresponding to a closed system with respect to the $X$ species (there is no exchange of internal species $X$ with the exterior). Evidently, the clamped system, Eq. (14), can be kept away from equilibrium by selecting the fixed concentrations $a_{u}$ of the external species, and this is tantamount to varying the rate constants $k_{w}^{\prime \pm}(\boldsymbol{a})$. Nevertheless, the clamped reaction rates in Eqs. (15) and (17) for the reversible transformations contribute exclusively to an entropy production $\sigma \geqslant 0$ [Eq. (2)] (per unit volume) but make no contribution to the entropy exchange (per unit volume) by volumetric flow $\sigma_{e}=0$ [Eq. (3)]. Note moreover that only diffusion fluxes $\boldsymbol{j}_{i}$ are included in their demonstration, but not matter fluxes, due to volumetric fluid flow exchange between the interior and the exterior.

The second point has to do with establishing the definiteness of the quadratic form in Eq. (11). For this, thermodynamic stability conditions are appealed to in order to establish a definite sign, in the above case, to ensure $\left(\frac{\partial \mu_{i}}{\partial \rho_{j}}\right)_{\text {eq }} \geqslant 0$. The sign is inferred from considering the second variation of the equilibrium thermodynamic potential $\left(\delta^{2} \phi_{v}\right)_{\mathrm{eq}} \geqslant 0$, Eq. (12). This is an equilibrium or pseudoequilibrium result, its validity in nonequilibrium thermodynamics follows of course from the assumption of local equilibrium [3]. In other demonstrations of the general evolution criterion, thermal and mechanical stability conditions have been invoked (positivity of specific heat at constant volume and of the isothermal compressibility) to ensure the definiteness of the quadratic forms, implied by the second variation of the equilibrium entropy $\left(\delta^{2} S\right)_{\mathrm{eq}} \leqslant 0[1,19,20]$.

\section{THE EVOLUTION THEOREM}

Consider chemical reactions for $r$ reactions $(j=1, \ldots, r)$ and $n$ reacting species obeying mass-action kinetics:

$$
\alpha_{1 j} X_{1}+\cdots+\alpha_{n j} X_{n} \stackrel{k_{j}}{\rightarrow} \beta_{1 j} X_{1}+\cdots+\beta_{n j} X_{n},
$$


where the $X_{i}, 1 \leqslant i \leqslant n$, are the species and $k_{j}>0$ is the reaction rate constant for the $j$ th reaction. Forward and reverse reactions are treated individually. Thus, Eq. (18) can also encompass any matter-flux terms we may want to include: Thus, $\stackrel{f\left[X_{u}\right]_{\text {in }}}{\rightarrow} X_{u}$ is written for a species flowing into the reactor with concentration $\left[X_{u}\right]_{\text {in }}$, and $X_{u} \stackrel{f}{\rightarrow}$ for outflow. A given species can flow out of the reactor (say, as a product) without necessarily flowing into it. A species flowing in at a specified input concentration can flow out with its instantaneous concentration, as established within the reactor. The entries of the $n \times r$ stoichiometric matrix $v_{i j}=\beta_{i j}-\alpha_{i j}$ are read off directly from Eq. (18). Note that our complete stoichiometric matrix $v$ includes the stoichiometric coefficients for both the internal reactions and the volumetric flow contributions, in contrast to that appearing in the mass-balance equation, Eq. (10), which accounts solely for the chemical reactions of the intermediate species.

The reaction rate $w_{j}$ corresponding to the $j$ th reaction takes the form of a monomial

$$
w_{j}\left(x, k_{j}\right)=k_{j} \prod_{i=1}^{n} x_{i}^{\alpha_{i j}}
$$

where $x_{i}=\left[X_{i}\right]$ are the concentrations. The chemical force, or affinity $A_{j}$ [3] of the $j$ th reaction, is given by de Donder's relation [3]

$$
A_{j}=-\sum_{k=1}^{n} v_{k j} \mu_{k}
$$

and $\mu_{k}$ is the chemical potential of the $k$ th species. Since the entropy production $\sigma$ is the product of a force times the flow it creates [1-3], we therefore consider the products of the affinities (the forces) times the reaction rates (the flows), summed over all the reversible reactions and the input-output volumetric flows. Using Eqs. (19) and (20), this works out to give (for details, see Appendix A:

$$
\sum_{k} J_{k}^{\text {react }} X_{k}^{\text {react }}+\sum_{i} J_{i}^{\text {flow }} X_{i}^{\text {flow }}=\sum_{j=1}^{r} w_{j}\left(\frac{A_{j}}{T}\right)=\sigma+\sigma_{e}
$$

where the force $X$ and corresponding flow $J$ for the volumetric matter flows are defined in Eqs. (A20) and (A21). This yields the entropy production plus the entropy exchange, where $T$ is the absolute temperature. This is the entropy balance equation, Eq. (1) (per unit volume).

An evolution criterion governs dynamics. We therefore consider how the temporal derivative of the forces (the affinities) can affect the balance equation (21) and so define the quantity $\Psi$ via

$$
\Psi=\sum_{j=1}^{r} w_{j} \frac{d}{d t}\left(\frac{A_{j}}{T}\right) .
$$

Having established the important identity of Eq. (21), (A24), we consider the calculating the sum over products of the reaction rates times the derivatives of the affinities.

We evaluate this directly by calculating the temporal derivative of the affinity from Eq. (20). The derivatives of the affinities, through their dependence on the chemical potentials
(20), involve the kinetic rate equations for the concentrations. The latter are expressed in terms of the complete stoichiometric matrix (internal reactions plus volumetric flows) and the corresponding reaction rates:

$$
\frac{d x_{k}}{d t}=\sum_{l=1}^{r} v_{k l} w_{l}
$$

The affinities are

$$
\begin{aligned}
A_{j} & =-\sum_{k=1}^{n} v_{k j} \mu_{k}^{r e l}, \\
& =-R T \sum_{k=1}^{n} v_{k j} \ln \left(\frac{x_{k}}{x_{k}^{\mathrm{eq}}}\right),
\end{aligned}
$$

where $\mu_{k}^{\text {rel }}$ is the relative chemical potential (4). Calculating the temporal derivative directly gives

$$
\begin{aligned}
\frac{d A_{j}}{d t} & =-R T \sum_{k=1}^{n} v_{k j} \frac{d}{d t} \ln \left(\frac{x_{k}}{x_{k}^{\mathrm{eq}}}\right), \\
& =-R T \sum_{k=1}^{n} v_{k j} \frac{1}{x_{k}}\left(\frac{d x_{k}}{d t}\right), \\
& =-R T \sum_{k=1}^{n} \frac{v_{k j}}{x_{k}}\left(\sum_{l=1}^{r} v_{k l} w_{l}\right), \\
& =-R T \sum_{l=1}^{r} \underbrace{\left(\sum_{k=1}^{n} \frac{v_{k j} v_{k l}}{x_{k}}\right)} w_{l} \leqslant 0 .
\end{aligned}
$$

The final inequality holds because the underbraced term is semipositive definite: That is, the sum over $k$ defines the $j l$ element of a Gram matrix [21], since $x_{k}>0$. Since the individual reaction rates $w_{l}>0$ are always positive, we are summing up semipositive definite contributions. Hence, the entire expression (because of the overall minus sign) is negative semidefinite. This proves that Eq. (29) is strictly negative semidefinite. Finally, inserting Eq. (29) into (22) immediately implies that $\Psi \leqslant 0$ (=0 on NESS), which is our thermodynamic evolution theorem:

$$
\Psi=\sum_{j=1}^{r} w_{j} \frac{d}{d t}\left(\frac{A_{j}}{T}\right) \leqslant 0 \quad(=0 \mathrm{NESS}) .
$$

Moreover, from Eqs. (21), (22), and (29) we also conclude

$$
\Psi=\frac{d_{A}\left(\sigma+\sigma_{e}\right)}{d t} \leqslant 0 \quad(=0 \mathrm{NESS}) .
$$

Here, $d_{A} / d t$ is the change with respect to the temporal derivative of the affinities. This is the statement that the chemical forces (the affinities $A_{j}$ ) must evolve in such a way as to lower the sum of the entropy production $\sigma$ and the entropy exchange $\sigma_{e}$. Note for the "clamped" approximation, the volumetric flow is absent, $q=f=0$, there is no exchange entropy $\sigma_{e}=0$, and our result in Eq. (31) then reduces to that of Glansdorff and Prigogine [1], who assumed both timeindependent boundary conditions and external forces [22]. The definite character of the quadratic form derived here (29) is a direct consequence of the linear-algebraic properties of products of the elements of this complete stoichiometric 
matrix. We include the volumetric open flow terms through the stoichiometric matrix $v_{i j}$, which accounts for all the transformations: Both the internal microreversible chemical transformations and the irreversible input-ouput flows in the well-mixed approximation.

From Eqs. (23), (29), and (30), we can express $\Psi$ as an inner product of a vector with itself:

$$
\Psi=-R \frac{d \boldsymbol{x}}{\sqrt{\boldsymbol{x}} d t} \cdot \frac{d \boldsymbol{x}}{\sqrt{\boldsymbol{x}} d t}=-R\left\|\frac{d \boldsymbol{x}}{\sqrt{\boldsymbol{x}} d t}\right\|^{2} \leqslant 0,
$$

where the vector components are $(k=1,2, \ldots, n)$

$$
\boldsymbol{v}_{k}=\left(\frac{d \boldsymbol{x}}{\sqrt{\boldsymbol{x}} d t}\right)_{k}=\sum_{l=1}^{r} \frac{v_{k l} w_{l}}{\sqrt{x_{k}}} .
$$

Then $\boldsymbol{v}_{k}$ is the differential rate equation $d x_{k} / d t$, divided by $\sqrt{x_{k}} \cdot \Psi$ is therefore invariant under rotations $\boldsymbol{v}_{j}^{\prime}=\boldsymbol{R}_{j k} \boldsymbol{v}_{k}$, for $\boldsymbol{R}$ an element of the rotation group in $n$ dimensions. Related symmetry properties are discussed in Appendix B, where $\Psi$ itself is expressed in terms of a Gram matrix.

A further important result follows from identifying the reaction pathways of the open-flow scheme. The matter fluxes traversing the network are identified using stoichiometric network analysis (SNA) [13]. Then, the hybrid pathwise entropy productions and exchanges can be calculated for each pathway, or elementary flux mode $\boldsymbol{E}_{i}(\mathrm{EFM})$, and the sum over all these $m$ modes, or pathways, obeys the identity [16,23]

$$
\sum_{i=1}^{m} \sigma\left(\boldsymbol{E}_{i}\right)=\sigma+\sigma_{e} \equiv V^{-1} \frac{d S}{d t},
$$

where $\boldsymbol{E}_{i}$ represents an elementary flux mode, or reaction pathway: It is made up from a specific sequence of transformations (18) that belong to the network. The $m$ vectors $\left\{\boldsymbol{E}_{i}\right\}_{i=1}^{m}$ have $r$ components, equal to the number of individual irreversible reactions (including the input-output flow terms) [13]. Then $\sigma\left(\boldsymbol{E}_{i}\right)$ denotes the weighted sum of the partial entropy productions and exchanges due to each of the transformations making up the $i$ th flux mode (see Sec VI). The identity Eq. (34) resolves the entropy balance equation (1) in terms of pathwise entropy production-exchange contributions. Thus, from (31) and (34) we conclude that

$$
\Psi=\frac{d_{A}}{d t} \sum_{i=1}^{m} \sigma\left(\boldsymbol{E}_{i}\right) \leqslant 0 \quad(=0 \mathrm{NESS}),
$$

which states that the affinities $A$ evolve in time such that the sum of the pathwise entropy productions and exchanges, taken over all the $m$ pathways of the network, is minimized. Furthermore, the dissipation of the complete network obeys a graded equipartition over the individual transformations that make up the elementary pathways. That is, a specific reaction in (18) that participates in $n$ different pathways will contribute a fraction $(1 / n)$ of its partial entropy production and/or exchange to each one of those pathways. This resolution (34) reveals those pathways for which the production and exchange of entropy are either maximized or minimized in compliance with the thermodynamic constraint (35); see Sec. VI for an explicit worked example.

Together, Eqs. (29), (31), and (35) are the main results of this paper. We have incorporated volumetric continuous flow terms, which give rise to an entropy exchange $\sigma_{e}$ which must be included with the entropy production $\sigma \geqslant 0$. Both terms are necessarily involved in the generalization of the GEC to volumetric open flow architectures. Continuous open flows are represented by flux terms [16], when integrated over the system boundary surface, give rise to the expression for $\sigma_{e}$, Eq. (3), in the approximation of instant diffusion and perfect mixing. The original derivations of the GEC $[1,19]$ were based on the assumption of local thermodynamic stability, used the negativity of the second-order variation of the equilibrium entropy $\delta^{2} S_{\text {eq }}<0$, and carried this over to nonequilibrium settings $[1,3]$. Our demonstration that $\Psi \leqslant 0$ follows directly from the fact that the stoichiometric matrix $v_{i j}$ enters quadratically in the time derivative of the chemical affinities, Eq. (29), yielding a structure recognized to be a positive definite Gram matrix. Note that

$$
\left(\frac{\partial \mu_{i}}{\partial x_{j}}\right)_{\mathrm{eq}}=\frac{R T}{x_{i}} \delta_{i j} \geqslant 0,
$$

proving that the inequality established in Eq. (29) is a consequence of the thermodynamic stability of the ideal solution.

\section{ENTROPIC ANALYSIS IN SPONTANEOUS MIRROR SYMMETRY BREAKING}

We calculate the entropy production and the exchange entropy and validate the thermodynamic evolution theorem Eq. (31) as well as analyze the individual pathwise entropy productions $\sigma\left(\boldsymbol{E}_{i}\right)$ in the context of spontaneous mirror symmetry breaking [14]. For this purpose, we will employ the Frank model, Fig. 1, which undergoes a bifurcation from an initially metastable racemic, or mirror-symmetric, state to one of two energetically degenerate stable scalemic states: See Fig. 2. There is a relatively short induction period in which both the enantiomers $L, D$ remain close to their initial concentrations, followed by a burst in their joint concentrations and consumption of the achiral resource $A$. This is followed by a rapid increase in the production of the achiral heterodimer $P$, with a corresponding drop in the still joint concentrations of $L, D$ to a very low level. The high level of heterodimer and the low levels of the enantiomers become unstable, provoking the bifurcation in the enantiomeric concentrations and a drop in the heterodimer concentration. Beyond this point, the system remains in this stable NESS. In this simulation example, we have chosen a slight initial excess for the concentration in the $L$ enantiomer over that of the $D$. This initially minuscule chiral fluctuation, equivalent to an initial enantiomeric excess $e e_{0}=$ $5.0 \times 10^{-18}$, suffices to take the system out from the unstable racemic NESS and evolves toward the stable scalemic NESS, resulting in an amplification of the initial ee to nearly $100 \%$ homochirality; see Fig. 2. The Frank model (see Appendix C) has been implemented experimentally by suitable chemical reactions [24]. The calculation of the pathwise entropy productions $\sigma\left(\boldsymbol{E}_{i}\right)$, described below in detail in Sec. VI, provides additional specific insight into the mirror symmetry breaking transition in terms of the dissipations along each pathway.

The entropy production sheds light on both stability properties [1,3] and on mirror symmetry breaking transitions [25-28] in chemical systems. These situations are characterized by transitions from metastable racemic to stable scalemic 

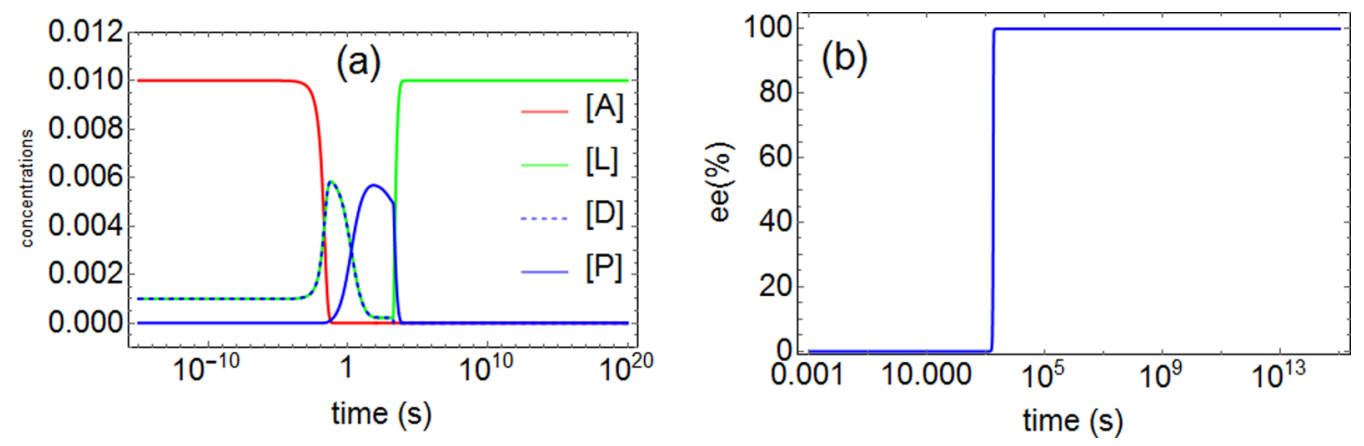

FIG. 2. Spontaneous mirror symmetry breaking (SMSB) for the Frank model in Fig. 1 (see also Appendix C). (a) Evolution of the concentrations of the achiral resource $A$, the enantiomers $L$ and $D$, and the heterodimer $P$. (b) Percent enantiomeric excess ee $(\%)=([L]-$ $[D]) /([L]+[D]) \times 100 \%$. Rate constants: $k_{a}=1 \times 10^{4}, k_{-a}=1 \times 10^{-4}, k_{d}=1 \times 10^{-2}, k_{-d}=1 \times 10^{-10}, k_{2}=1 \times 10^{2}, k_{-2}=1 \times 10^{-4} ;$ initial conditions $(\mathrm{mol} / \mathrm{L}):[A]_{0}=[A]_{\text {in }}=1 \times 10^{-2},[L]_{0}=1 \times 10^{-3}+1 \times 10^{-20},[D]_{0}=1 \times 10^{-3}-1 \times 10^{-20},[P]_{0}=1 \times 10^{-6}$.

nonequilibrium stationary states (NESS), respectively. Figure 3 shows the evolution of the entropy production per unit volume $\sigma \geqslant 0$, Eq. (2), and its subsequent minimization in the final stable chiral state relative to the racemic; the entropy balance $d S / d t$, Eq. (1), evolves to zero: This is confirmed since $\sigma_{e}=-\sigma<0$ in the stationary chiral state (Fig. 3 and see also Fig. 5). In Fig. 4, the change in entropy production with respect to the time derivative of the affinities, $d_{A} \sigma / d t$, is negative and goes to zero from below on the approach to the final stable chiral NESS; the change in the entropy exchange $d_{A} \sigma_{e} / d t$ is positive and goes to zero at the NESS (Fig. 4). Summing both these separate contributions obeys the evolution criterion $\Psi \leqslant 0$, Eq. (31), and is negative, approaching zero asymptotically from below, as the system settles down into the final stable chiral state; see Fig. 5.

\section{PATHWAY ENTROPY PRODUCTION AND EXCHANGE}

We consider the partial entropy productions and exchanges [16] associated with each individual unidirectional transformation (see Appendix C) in Fig. 1. These are defined and calculated in Table I for the chemical transformations and volumetric flow terms involved. Their weighted sums yield the combined, or hybrid, entropy production and exchange $\sigma\left(\boldsymbol{E}_{i}\right)$ along each pathway or elementary flux mode $\boldsymbol{E}_{i}(\mathrm{EFM})$, and the sum over all the $m$ pathways or EFMs obeys the entropy balance equation (34) and, hence from Eq. (31), thus also the

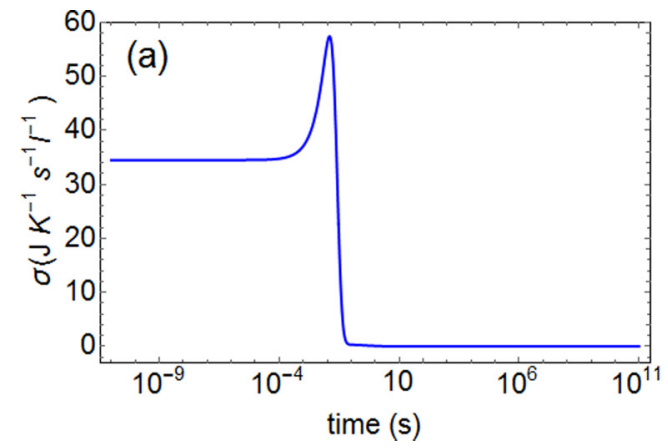

thermodynamic evolution criterion (35). The open-flow Frank model, Fig. 1, has $m=18$ EFMs, 14 of which correspond to seven enantiomeric pairs or doublets under $L \leftrightarrow D$ interchange, while the remaining four EFMs are mirror-symmetric singlets; see Table II. The EFMs are defined and calculated using stoichiometric network analysis (SNA) in Appendix C 1 and are listed in Table II, indicating the sequence of transformations each EFM represents.

From Tables I and II, we define and calculate the entropy production and entropy exchange along each pathway, or along each extreme flux mode (EFM) $\boldsymbol{E}_{i}$, as follows. We first calculate the partial entropy production or exchange corresponding to each individual irreversible transformation (using Table I) and then form the weighted sum of these partial contributions along the flux mode $\boldsymbol{E}_{i}$ to define the entropy production and exchange along each reaction pathway. For the $r$ th transformation, the inverse of the number of nonzero entries in the $r$ th row of the matrix $\boldsymbol{E}$ in Eq. (C17) provides the relative weight, which must be included to avoid double counting. Carrying out this procedure leads to

$$
\begin{gathered}
\sigma\left(\boldsymbol{E}_{1}\right)=\frac{1}{5} \sigma(L+D \rightarrow P)+\sigma(P \rightarrow L+D), \\
\sigma\left(\boldsymbol{E}_{2}\right)=\frac{1}{5} \sigma(A \rightarrow D)+\frac{1}{2} \sigma(D \rightarrow A), \\
\sigma\left(\boldsymbol{E}_{3}\right)=\frac{1}{5} \sigma(A+D \rightarrow 2 D)+\frac{1}{2} \sigma(D \rightarrow A),
\end{gathered}
$$

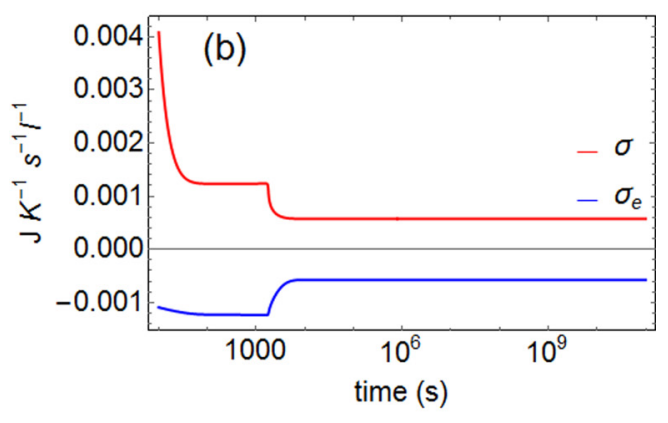

FIG. 3. (a) The entropy production $\sigma>0$ for the Frank model in Fig. 1 over the time range of the simulation (see also Appendix C). (b) The entropy production $\sigma \geqslant 0$ and exchange entropy $\sigma_{e}$ on their approach to the final chiral nonequilibrium stationary state where $\sigma_{e}=-\sigma<0$ for the NESS. This confirms that $V^{-1} d S / d t=\sigma+\sigma_{e}=0$ vanishing on the stationary states. 

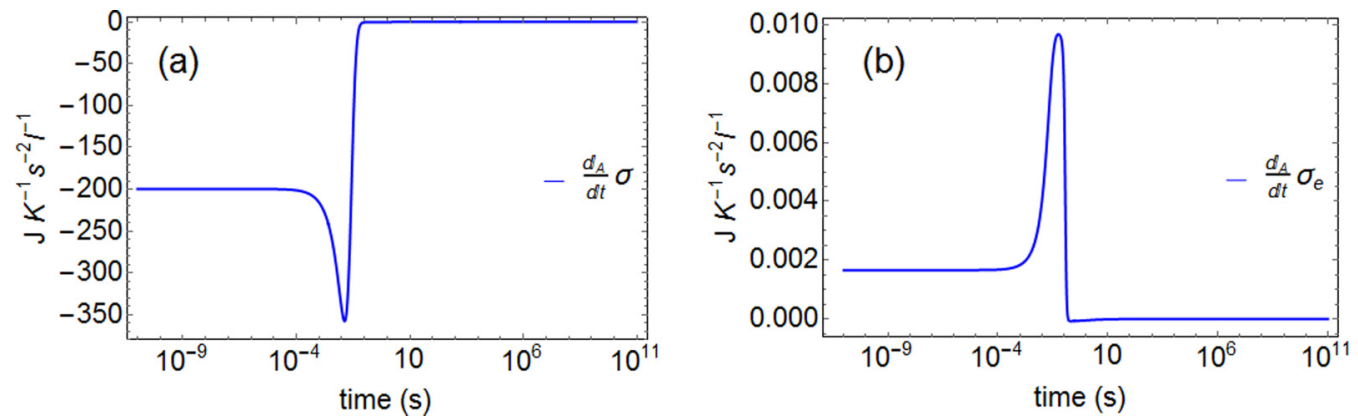

FIG. 4. (a) The rate of change of the entropy production per unit volume $d_{A} \sigma / d t$ with respect to the time derivative of the affinities. (b) The change in the entropy exchange $d_{A} \sigma_{e} / d t$ with respect to the time derivative of the affinities. The sum of both quantities yields $\Psi=d_{A}\left(\sigma+\sigma_{e}\right) / d t \leqslant 0$; see Fig. 5 .

$$
\begin{gathered}
\sigma\left(\boldsymbol{E}_{4}\right)=\frac{1}{2} \sigma(2 D \rightarrow A+D)+\frac{1}{5} \sigma(A \rightarrow D), \\
\sigma\left(\boldsymbol{E}_{5}\right)=\frac{1}{5} \sigma(A+D \rightarrow 2 D)+\frac{1}{2} \sigma(2 D \rightarrow A+D), \\
\sigma\left(\boldsymbol{E}_{6}\right)=\frac{1}{5} \sigma(A \rightarrow L)+\frac{1}{2} \sigma(L \rightarrow A), \\
\sigma\left(\boldsymbol{E}_{7}\right)=\frac{1}{5} \sigma(A+L \rightarrow 2 L)+\frac{1}{2} \sigma(L \rightarrow A), \\
\sigma\left(\boldsymbol{E}_{8}\right)=\frac{1}{2} \sigma(2 L \rightarrow A+L)+\frac{1}{5} \sigma(A \rightarrow L), \\
\sigma\left(\boldsymbol{E}_{9}\right)=\frac{1}{5} \sigma(A+L \rightarrow 2 L)+\frac{1}{2} \sigma(2 L \rightarrow A+L), \\
\sigma\left(\boldsymbol{E}_{10}\right)=\frac{1}{9} \sigma(\rightarrow A)+\sigma(A \rightarrow), \\
\sigma\left(\boldsymbol{E}_{11}\right)=\frac{1}{9} \sigma(\rightarrow A)+\frac{1}{5} \sigma(A \rightarrow D)+\frac{1}{2} \sigma(D \rightarrow) \\
\sigma\left(\boldsymbol{E}_{13}\right)=\frac{1}{9} \sigma(\rightarrow A)+\frac{1}{5} \sigma(A \rightarrow L)+\frac{1}{2} \sigma(L \rightarrow) \\
\sigma\left(\boldsymbol{E}_{14}\right)=\frac{1}{9} \sigma(\rightarrow A)+\frac{1}{5} \sigma(A+L \rightarrow 2 L)+\frac{1}{2} \sigma(L \rightarrow), \\
\sigma\left(\boldsymbol{E}_{15}\right)=\frac{1}{9} \sigma(\rightarrow A)+\frac{1}{5} \sigma(A \rightarrow L)+\frac{1}{5} \sigma(A \rightarrow D) \\
+\frac{1}{5} \sigma(L+D \rightarrow P)+\frac{1}{4} \sigma(P \rightarrow)
\end{gathered}
$$

$$
\begin{aligned}
\sigma\left(\boldsymbol{E}_{16}\right)= & \frac{1}{9} \sigma(\rightarrow A)+\frac{1}{5} \sigma(A \rightarrow L)+\frac{1}{5} \sigma(A+D \rightarrow 2 D) \\
& +\frac{1}{5} \sigma(L+D \rightarrow P)+\frac{1}{4} \sigma(P \rightarrow), \\
\sigma\left(\boldsymbol{E}_{17}\right)= & \frac{1}{9} \sigma(\rightarrow A)+\frac{1}{5} \sigma(A \rightarrow D)+\frac{1}{5} \sigma(A+L \rightarrow 2 L) \\
& +\frac{1}{5} \sigma(L+D \rightarrow P)+\frac{1}{4} \sigma(P \rightarrow), \\
\sigma\left(\boldsymbol{E}_{18}\right)= & \frac{1}{9} \sigma(\rightarrow A)+\frac{1}{5} \sigma(A+L \rightarrow 2 L)+\frac{1}{5} \sigma(A+D \rightarrow 2 D) \\
& +\frac{1}{5} \sigma(L+D \rightarrow P)+\frac{1}{4} \sigma(P \rightarrow) .
\end{aligned}
$$

The relative fractional weights reflect the fact that the partial entropy production and exchange corresponding to each elementary irreversible transformation (see Table I) is equipartitioned over the number of EFMs $\boldsymbol{E}_{i}$ in which that specific transformation participates. For example, the partial entropy production $\sigma(A+D \rightarrow 2 D)$ contributes a fraction $1 / 5$ of its magnitude to each one of the five distinct EFMs that involve this catalytic step, $\boldsymbol{E}_{3}, \boldsymbol{E}_{5}, \boldsymbol{E}_{12}, \boldsymbol{E}_{16}$, and $\boldsymbol{E}_{18}$; see Table II. Summing up all these partial entropy productions and exchanges over the set of 18 EFM's, and using Table I, it is straightforward to verify that

$$
\sum_{i=1}^{18} \sigma\left(\boldsymbol{E}_{i}\right)=\sigma+\sigma_{e}=V^{-1} \frac{d S}{d t}
$$

where $\sigma \geqslant 0$ is the entropy production and $\sigma_{e}$ is the entropy exchange, per unit volume; see Eqs. (2) and (3). This result, Eq. (55), resolves the sum of the entropy production and
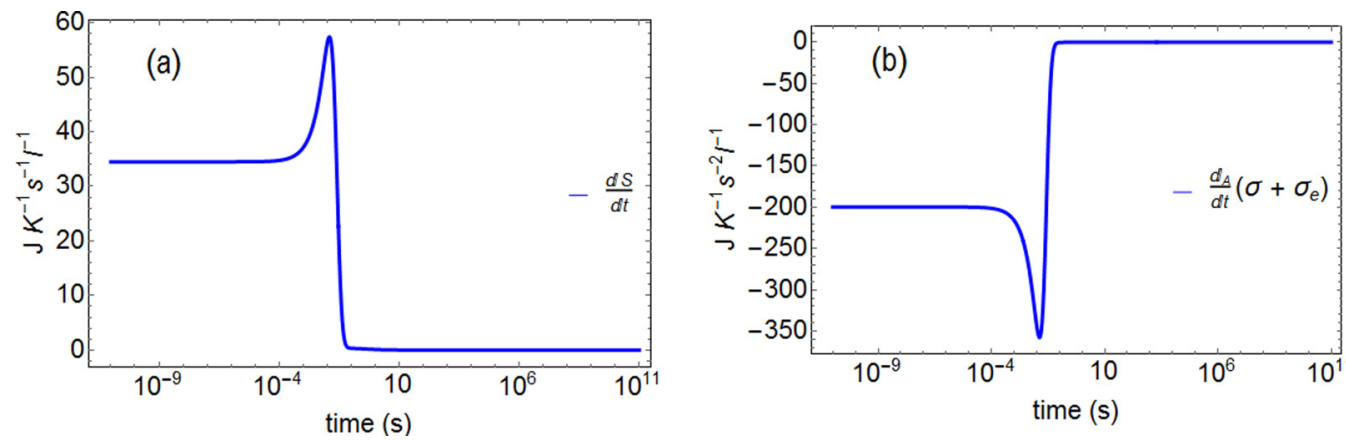

FIG. 5. (a) The time rate of change of the system entropy per unit volume $V^{-1} d S / d t=\sigma+\sigma_{e}$. (b) The change in the entropy production and exchange entropy with respect to the time derivative of the affinities $\Psi=d_{A}\left(\sigma+\sigma_{e}\right) / d t \leqslant 0$, and obeys our thermodynamic evolution criterion, Eq. (31), for the volumetric open-flow reaction scheme in Fig. 1. 
TABLE I. The partial entropy productions and entropy exchanges (per unit volume) for the transformations involved in the open-flow Frank scheme: $(\mathrm{C} 1)-(\mathrm{C} 8)$.

\begin{tabular}{|c|c|}
\hline Transformation: & Partial entropy production or exchange (per unit volume) \\
\hline$A+L \stackrel{k_{a}}{\longrightarrow} 2 L$ & $\sigma(A+L \rightarrow 2 L)=R k_{a}[A][L] \ln \left(\frac{k_{a}[A]}{k_{-a}[L]}\right)$ \\
\hline $2 L \stackrel{k_{-a}}{\longrightarrow} A+L$ & $\sigma(2 L \rightarrow A+L)=R k_{-a}[L]^{2} \ln \left(\frac{k_{-a}[L]}{k_{a}[A]}\right)$ \\
\hline$A \stackrel{k_{d}}{\longrightarrow} L$ & $\sigma(A \rightarrow L)=R k_{d}[A] \ln \left(\frac{k_{d}[A]}{k_{-d}[L]}\right)$ \\
\hline$L \stackrel{k_{-d}}{\longrightarrow} A$ & $\sigma(L \rightarrow A)=R k_{-d}[L] \ln \left(\frac{k_{-d}[L]}{k_{d}[A]}\right)$ \\
\hline$L+D \stackrel{k_{1}}{\longrightarrow} P$ & $\sigma(L+D \rightarrow P)=R k_{1}[L][D] \ln \left(\frac{k_{1}[L][D]}{k_{-1}[P]}\right)$ \\
\hline$P \stackrel{k_{-1}}{\longrightarrow} L+D$ & $\sigma(P \rightarrow L+D)=R k_{-1}[P] \ln \left(\frac{k_{-1}[P]}{k_{1}[L][D]}\right)$ \\
\hline$\stackrel{f[A]_{\text {in }}}{\longrightarrow} A$ & $\sigma(\rightarrow A)=R f[A]_{\mathrm{in}} \ln \left(\frac{[A]_{\mathrm{eq}}}{[A]}\right)$ \\
\hline$Y \stackrel{f}{\longrightarrow}$ & $\sigma(Y \rightarrow)=R f[Y] \ln \left(\frac{[Y]}{[Y] \mathrm{eq}}\right)$ \\
\hline
\end{tabular}

exchange entropy $\sigma+\sigma_{e}$ in terms of the (hybrid) pathway entropy contributions $\sigma\left(\boldsymbol{E}_{i}\right)$, summed up over all the pathways. This is the entropy balance equation, Eq. (1), expressed in terms of the pathway entropy productions and exchanges associated with each individual EFM.

Consider first the hybrid entropy production and exchange for the enantiomeric pathway pair $\boldsymbol{E}_{16}, \boldsymbol{E}_{17}$. These are expressed as weighted sums over the partial entropy productions and/or exchanges associated with each individual transformations as indicated; the fractional weights follow from stoichiometric network analysis [13]. These pathways involve the (i) input of A to the reactor, (ii) the direct conversion of $A$ to enantiomers $L$ or $D$, (iii) enantioselective autocatalysis, (iv) followed by mutual inhibition (formation of the heterodimer $P$ ), and then (v) the outflow of the product $P$ from the reactor. The mirror symmetry of the pathway pair,
Eqs. (52) and (53), under interchange of $L \leftrightarrow D$ is manifest. Nevertheless, the evolution of the system leads to a mirror symmetry breaking bifurcation in this, as well in all seven pairs of pathway entropy productions corresponding to the 14 enantiomeric extreme flux modes; see Fig. 6 for two characteristic examples. After symmetry breaking, we find $\sigma\left(\boldsymbol{E}_{17}\right)>\sigma\left(\boldsymbol{E}_{16}\right)$ and $\sigma\left(\boldsymbol{E}_{14}\right)>\sigma\left(\boldsymbol{E}_{12}\right)$. The distinguishing chemical feature in the first pathway pair is the combination of the direct productions $A \rightarrow L(D)$ and the enantioselective autocatalyses $A+D(L) \rightarrow 2 D(L)$; in the latter pair, it is the combination of the autocatalyses and the outflows of each enantiomer (Fig. 6).

Mirror symmetry breaking leads to bifurcations in the values of the pathway entropy productions and exchanges associated with the mirror-symmetric related EFM pair $\sigma\left(\boldsymbol{E}_{2}\right)>\sigma\left(\boldsymbol{E}_{6}\right)$ and also with the pair $\sigma\left(\boldsymbol{E}_{7}\right)>\sigma\left(\boldsymbol{E}_{3}\right)$,

TABLE II. Frank open-flow scheme (see Fig. 1): the 18 elementary flux modes (EFM) $\boldsymbol{E}_{i}$, the individual transformations they involve as enumerated in Eqs. (C1)-(C8), and their corresponding reaction pathways. These currents can be grouped into a subset of seven mirror symmetric doublets under $L \leftrightarrow D$ interchange: $\boldsymbol{E}_{2} \Leftrightarrow \boldsymbol{E}_{6}, \boldsymbol{E}_{3} \Leftrightarrow \boldsymbol{E}_{7}, \boldsymbol{E}_{4} \Leftrightarrow \boldsymbol{E}_{8}, \boldsymbol{E}_{5} \Leftrightarrow \boldsymbol{E}_{9}, \boldsymbol{E}_{11} \Leftrightarrow \boldsymbol{E}_{13}, \boldsymbol{E}_{12} \Leftrightarrow \boldsymbol{E}_{14}$, and $\boldsymbol{E}_{16} \Leftrightarrow \boldsymbol{E}_{17}$ and a subset of four mirror symmetric singlets: $\boldsymbol{E}_{1}, \boldsymbol{E}_{10}, \boldsymbol{E}_{15}, \boldsymbol{E}_{18}$. This is a $Z_{2}$ symmetry in composition space. See also Appendix C 1 .

\begin{tabular}{lll}
\hline \hline EFM: & Reactions & Subnetwork: reaction pathway \\
\hline $\boldsymbol{E}_{1}$ & $(9),(10)$ & $L+D \rightarrow P, P \rightarrow L+D$ \\
$\boldsymbol{E}_{2}$ & $(7),(8)$ & $A \rightarrow D, D \rightarrow A$ \\
$\boldsymbol{E}_{3}$ & $(5),(8)$ & $A+D \rightarrow 2 D, D \rightarrow A$ \\
$\boldsymbol{E}_{4}$ & $(6),(7)$ & $2 D \rightarrow A+D, A \rightarrow D$ \\
$\boldsymbol{E}_{5}$ & $(5),(6)$ & $A+D \rightarrow 2 D, 2 D \rightarrow A+D$ \\
$\boldsymbol{E}_{6}$ & $(3),(4)$ & $A \rightarrow L, L \rightarrow A$ \\
$\boldsymbol{E}_{7}$ & $(1),(4)$ & $A+L \rightarrow 2 L, L \rightarrow A$ \\
$\boldsymbol{E}_{8}$ & $(2),(3)$ & $2 L \rightarrow A+L, A \rightarrow L$ \\
$\boldsymbol{E}_{9}$ & $(1),(2)$ & $A+L \rightarrow 2 L, 2 L \rightarrow A+L$ \\
$\boldsymbol{E}_{10}$ & $(11),(12)$ & $\rightarrow A, A \rightarrow A, A \rightarrow D, D \rightarrow$ \\
$\boldsymbol{E}_{11}$ & $(11),(7),(14)$ & $\rightarrow A, A+D \rightarrow 2 D, D \rightarrow$ \\
$\boldsymbol{E}_{12}$ & $(11),(5),(14)$ & $\rightarrow A, A \rightarrow L, L \rightarrow$ \\
$\boldsymbol{E}_{13}$ & $(11),(3),(13)$ & $\rightarrow A, A+L \rightarrow 2 L, L \rightarrow$ \\
$\boldsymbol{E}_{14}$ & $(11),(1),(13)$ & $\rightarrow A, A \rightarrow L, A \rightarrow D, L+D \rightarrow P, P \rightarrow$ \\
$\boldsymbol{E}_{15}$ & $(11),(3),(7),(9),(15)$ & $\rightarrow A, A \rightarrow L, A+D \rightarrow 2 D, L+D \rightarrow P, P \rightarrow$ \\
$\boldsymbol{E}_{16}$ & $(11),(3),(5),(9),(15)$ & $\rightarrow A, A+L \rightarrow 2 L, A \rightarrow D, L+D \rightarrow P, P \rightarrow$ \\
$\boldsymbol{E}_{17}$ & $(11),(1),(7),(9),(15)$ & $\rightarrow A, A \rightarrow 2 L, A+D \rightarrow 2 D, L+D \rightarrow P, P \rightarrow$ \\
$\boldsymbol{E}_{18}$ & $(11),(1),(5),(9),(15)$ &
\end{tabular}



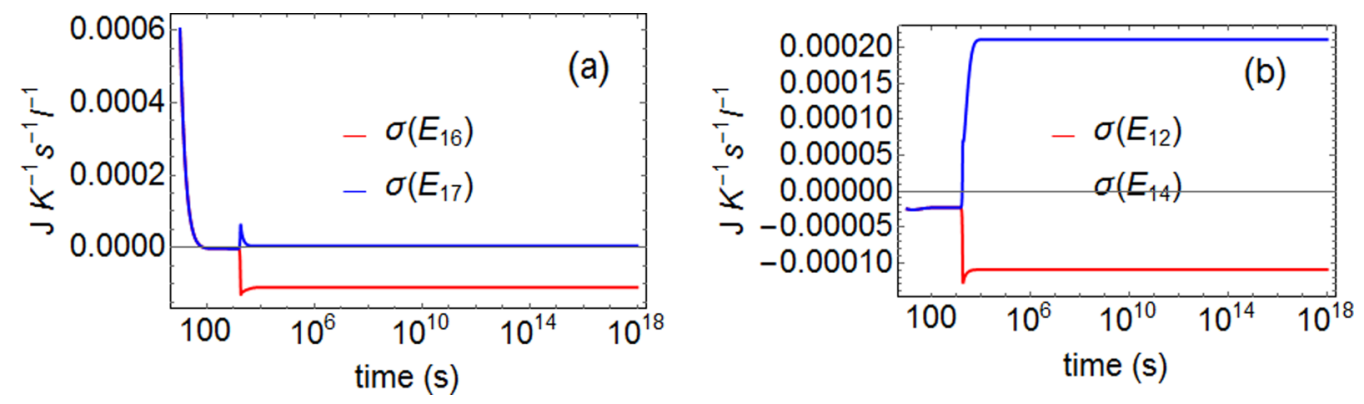

FIG. 6. Mirror symmetry breaking bifurcation in the pathway and hybrid entropy productions and/or exchange in the enantiomeric pairs of extreme flux modes $(\mathrm{EFM})$. (a) Bifurcation in the pair $\sigma\left(\boldsymbol{E}_{16}\right), \sigma\left(\boldsymbol{E}_{17}\right)$, Eqs. (52) and (53). (b) Bifurcation in the pair $\sigma\left(\boldsymbol{E}_{12}\right)=\frac{1}{9} \sigma(\rightarrow A)+\frac{1}{5} \sigma(A+D \rightarrow 2 D)+\frac{1}{2} \sigma(D \rightarrow)$ and $\sigma\left(\boldsymbol{E}_{14}\right)=\frac{1}{9} \sigma(\rightarrow A)+\frac{1}{5} \sigma(A+L \rightarrow 2 L)+\frac{1}{2} \sigma(L \rightarrow)$, Eqs. (48) and (50).

respectively; see Fig. 7. From Table II, $\boldsymbol{E}_{2}$ and $\boldsymbol{E}_{6}$ represent the pathways $A \rightarrow D, D \rightarrow A$, and $A \rightarrow L, L \rightarrow A$, respectively, the forward and inverse direct production of the enantiomers. Likewise, $\boldsymbol{E}_{7}$ and $\boldsymbol{E}_{3}$ represent the pathways $A+$ $L \rightarrow 2 L, L \rightarrow A$ and $A+D \rightarrow 2 D, D \rightarrow A$, respectively: the enantioselective catalyses and the decay of the enantiomers back into the achiral source.

Similarly, the pairs of pathway entropies associated with the following pathways show $\sigma\left(\boldsymbol{E}_{4}\right)>\sigma\left(\boldsymbol{E}_{8}\right)$ and $\sigma\left(\boldsymbol{E}_{9}\right)>$ $\sigma\left(\boldsymbol{E}_{5}\right)$, respectively, they undergo bifurcations: See Fig. 8. The first pathway pair involves the reverse catalyses plus the forward direct productions; see Table II. The second pathway pair are defined by the forward and reverse catalyses only. Figure 9 shows the bifurcation for the enantiomeric pathways $\sigma\left(\boldsymbol{E}_{13}\right)>\sigma\left(\boldsymbol{E}_{11}\right)$. These latter pathways involve the input of $A$, the direct production of enantiomer, followed by the exit of that same enantiomer from the reactor; see Table II.

For these initially mirror symmetric pathway entropy productions and exchanges, after SMSB the larger combined production plus exchange is sometimes along the pathways involving only the $L$-enantiomer and achiral source, as is the case for $\boldsymbol{E}_{7}, \boldsymbol{E}_{9}$, and $\boldsymbol{E}_{13}$, whereas the greater magnitude is sometimes for the pathways $\boldsymbol{E}_{2}$ and $\boldsymbol{E}_{4}$, which involve only the $D$ enantiomer and achiral source. This result may result counterintuitive, since we perturbed the initial racemic state with a slight excess in favor of the $L$ enantiomer. The pathway analysis therefore shows that, while the enantiomeric excess is indeed in the $L$ composition (see Fig. 2), the pathwise distributions of the overall entropy production and exchange can be maximized or minimized for some of the pathways involving only the minority enantiomer (in the case, the $D$ ), with respect to the majority enantiomer (in this case, the $L$ ).

From this EFM analysis, the reason why entropy production is minimized, with respect to the unstable racemic, in the case of SMSB [3,23,26-31] is intuitively clear: Whereas in the racemic state the pairs of EFMs in each $Z_{2}$ doublet dissipate identically, after SMSB the dissipation is diminished in one half of the $Z_{2}$ related doublet EFMs, while it is augmented slightly in the other half of the EFMs. This compositional $Z_{2}$ symmetry is broken, and this impacts directly on the overall system's dissipation and entropy exchange. We see this clearly from the bifurcations in the pathway entropy productions and entropy exchanges for the EFM doublets: Figs. 6-9.

The sum of the partial entropy productions and exchanges taken over all the extreme flux modes vanishes identically at any NESS, confirming that production and exchange over all such pathways are perfectly balanced at the final chiral NESS, as well as at the prior metastable racemic state, in compliance with Eq. (35); see also Fig. 5.

\section{DISCUSSION}

Stoichiometric network analysis (SNA) applied to a reaction network, in a system possessing input and output matter fluxes, yields the inequality for $\Psi$, Eq. (31), that is, the statement that the change in $\sigma+\sigma_{e}$ with respect to the time derivatives of the affinities $A$ is such as to lower the value of $d S / d t$ and which is zero in a stationary state. Thus, the chemical forces (the affinities) of the entire system as a whole evolve in time so as to lower the rate of change in the system entropy.
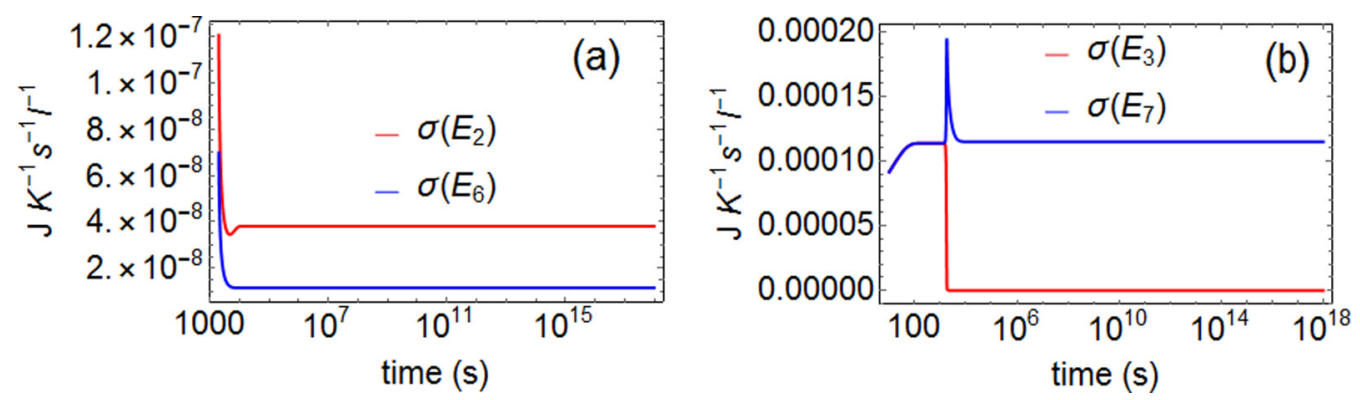

FIG. 7. Mirror symmetry breaking bifurcation in the pathway and hybrid entropy productions and/or exchanges corresponding to the enantiomeric pairs of extreme flux modes (EFM) in Table II. (a) Bifurcation in the pair $\sigma\left(\boldsymbol{E}_{2}\right), \sigma\left(\boldsymbol{E}_{6}\right)$; see Eqs. (38) and (42). (b) Bifurcation in the pair $\sigma\left(\boldsymbol{E}_{3}\right)$ and $\sigma\left(\boldsymbol{E}_{7}\right)$; see Eqs. (39) and (43). 

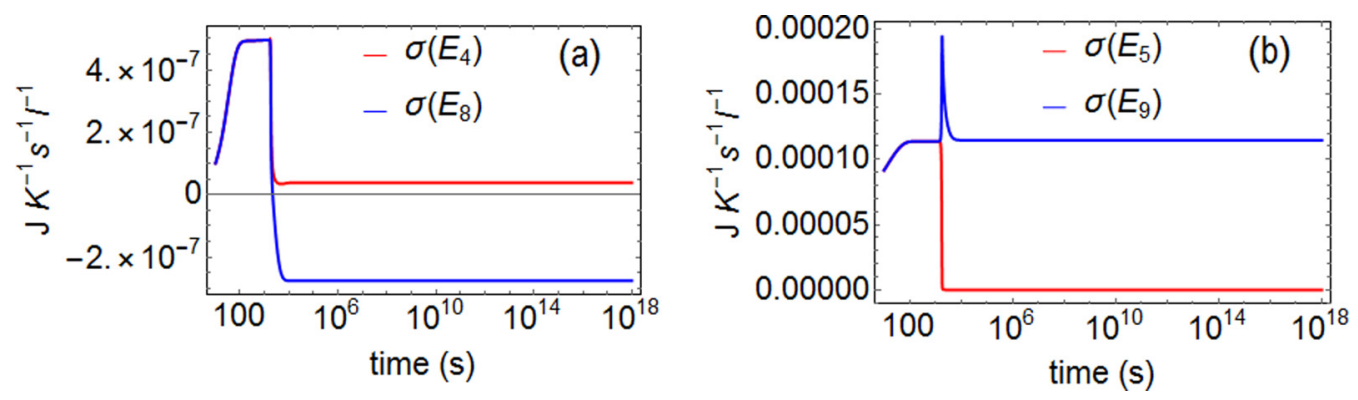

FIG. 8. Mirror symmetry breaking bifurcation in the pathway and hybrid entropy productions and/or exchanges corresponding to the enantiomeric pairs of extreme flux modes (EFM) in Table II. (a) Bifurcation in the pair $\sigma\left(\boldsymbol{E}_{4}\right), \sigma\left(\boldsymbol{E}_{8}\right)$; see Eqs. (40) and (44). (b) Bifurcation in the pair $\sigma\left(\boldsymbol{E}_{5}\right)$ and $\sigma\left(\boldsymbol{E}_{9}\right)$; see Eqs. (41) and (45).

This is a generalization of the general evolution criterion (GEC). Note, for the clamped approximation, when all the external concentrations are held at predetermined fixed values, these can be absorbed into the reaction rate constants $k$ of the internal reversible reactions. There is then no exchange entropy, $\sigma_{e}=0$, and our result reduces, as it must, to the standard GEC as proven originally by Glansdorff and Prigogine [1]. In their proof, they derived the GEC for dissipative processes involving a variety of generalized forces $X_{\alpha}$ which includes the chemical affinity. They state that " $[t]$ he change of the forces $X_{\alpha}$ proceeds always in a way as to lower the value of the entropy production" (p. 111) [1]. The original GEC demonstration follows as a consequence of the second law, but the inequality Eq. (31) for $\Psi$, in which the elements of the stoichiometric matrix define a Gram matrix in Eq. (29), necessarily gives a positive sign (negative, when the definition of de Donder's affinity is taken into account). This manifestly algebraic conclusion is a consequence of the ability of SNA to describe, through the stoichiometric matrix, coupled reaction networks in open systems: Each species's chemical potential shows a specific value, but this unique value determines positive or negative forces depending on the specific unidirectional reaction where it is acting. Notice that the correlation between the directions (that is, parallel or antiparallel) of the force $X_{\alpha}$ and the current $J_{\alpha}$, for example, between a temperature gradient and heat flow, or chemical potential difference and

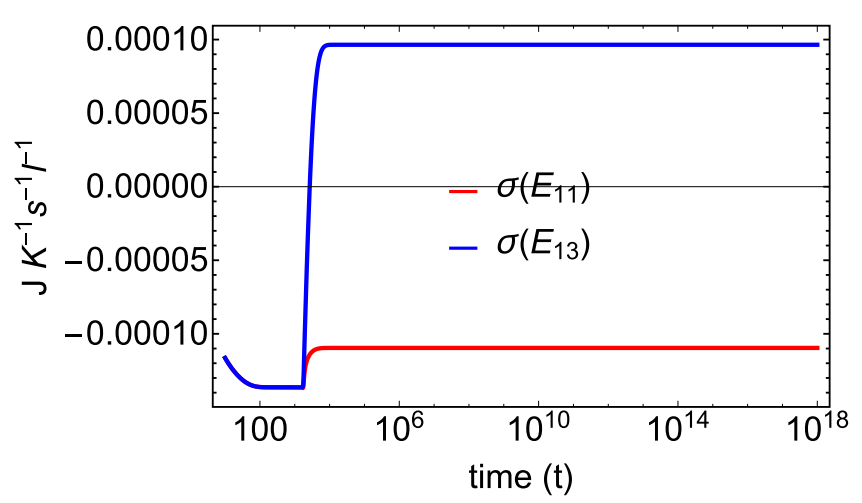

FIG. 9. Mirror symmetry breaking bifurcation in the pathway and hybrid entropy productions and/or exchanges corresponding to the enantiomeric pair of extreme flux modes $\sigma\left(\boldsymbol{E}_{11}\right)$ and $\sigma\left(\boldsymbol{E}_{13}\right)$; see Eqs. (47) and (49) and also Table II. absolute reaction rates, is a universal physical constraint prior to the second law. Therefore, $\Psi$ reveals this directional forcecurrent constraint.

Thermodynamic evolution of fluid flow-driven chemical reactions involves the internal processes taking place within the system as well as the matter fluxes into and out from the system (e.g., Fig. 1). The affinities must evolve to minimize the sum of the entropy production and entropy exchange. The set of reactions plus the input-output fluxes evolve jointly and in unison in compliance with this criterion Eq. (29), valid in the nonlinear and linear regimes of nonequilibrium thermodynamics, and vanishes for stationary states and for equilibrium. Application of SNA reveals that the (partial) entropy productions and exchanges associated with each individual irreversible reaction and matter flow term are equipartioned over those pathways in which those reactions and flow terms belong. The combined entropy productions and exchanges over each reaction pathway gives insight to the mechanisms involved in symmetry breaking. This result is important, for it links production of entropy and exchange entropy with pathways and hence with network topology. In this respect, open flow chemical systems have special interest regarding features of symmetry breaking in dynamical systems, as well as their obvious relevance for studying biochemical and biological problems in more involved and complex situations. Our results hold for macroscopic systems and ideal solutions. For sufficiently small systems, such as biological cells, stochastic nonequilibrium thermodynamics is likely required [32-35]. For this case, the mesoscopic approach leads to an evolution criterion in terms of the time derivative of the probability distribution [33] reminiscent of (29), where the latter is valid in the limit of zero fluctuations, and so our result can be regarded as the thermodynamic or deterministic limit of the former.

By making use of stoichiometric network analysis (SNA), we also derived an inequality constraining the pathway entropy productions associated to each extreme flux mode (EFM) and summed over all the EFMs. This representation of our thermodynamic inequality shows that the forces (the affinities of both the internal reactions and of the external matter fluxes) evolve in time so that the sum of the entropy productions and/or exchanges, taken over all the elementary flux modes $\boldsymbol{E}_{i}(\mathrm{EFM})$, of the network, is lowered. The latter result shows moreover how the entropy production and exchange gets shared out among all the individual reaction 
pathways of the system. An explicit set of EFMs is derived in Appendix C 1 for an open-flow version of the Frank model, and the inequality is validated for a mirror symmetry breaking transition. For the case of SMSB in the Frank model, the entropy production plus exchange entropy do reach a minimum value, with respect to the thermodynamic racemic branch after symmetry breaking.

At this juncture, it is important to emphasize, however, the fact that neither the original GEC nor our generalization to open flow, Eq. (31), necessarily imply that the entropy production (or the sum of the entropy production and the exchange entropy) will actually be minimized. This is because, beyond the linear regime of nonequilibrium thermodynamics, there is no general result governing the behavior of the changes in entropy production with respect to temporal derivatives of the flows $d_{J} \sigma / d t$, where the flows $J$ result from the generalized forces $X$. For the case of SMSB in the Frank model, the entropy production plus exchange entropy do reach a minimum value, with respect to the thermodynamic racemic branch after symmetry breaking. An interesting example, to contrast against our results for the Frank model, is provided by an autocatalytic enantioselective reaction scheme, without mutual inhibition, and with variable catalytic order $1<n \leqslant 2$, which can exhibit either bistability or even tristability, depending on the value of $n$. Then, for specified reaction rate and flow constants and for the range $1.6<n<1.7$, the model has one stable racemic NESS, one unstable scalemic NESS, and one stable scalemic NESS [36]. The corresponding entropy productions satisfy $\quad \sigma$ (stable racemic) $>\sigma$ (unstable scalemic) $>\sigma$ (stable scalemic) $>0$. Thus, if the system starts off initially in the unstable scalemic NESS, the sign of a random fluctuation will determine whether the system ends up in the stable racemic NESS or else in the stable scalemic NESS: In the latter case, the entropy production will be minimized, but in the former it will be maximized, with respect to that of the initial unstable scalemic state. Nevertheless, the thermodynamic inequality Eq. (31) is strictly obeyed for all the possible allowed outcomes [36].

In summary, we have derived an inequality which governs the rate of change of the entropy balance (the entropy production $\sigma \geqslant 0$ plus the exchange entropy $\sigma_{e}$ ) in volumetric open-flow chemical systems. This result governs the joint evolution of both the internal reactions taking place within the system and the matter fluxes that the system exchanges with its environment. It furthermore describes how the equipartition of the energy, over the reaction pathways belonging to the entire system, leads to a partitioning of the entropy production over the set of pathways with weights determined by the stoichiometry. The power (per unit volume) is distributed over the reaction paths and reveals a universal evolution criterion for extended nonlinear thermodynamics. The result is invariant under rotations corresponding to redefinitions of products of stoichiometric coefficients with reaction rate velocities. We validate our inequality for an enantioselective autocatalytic model possessing nonequilibrium stationary states (NESS) that lie off the thermodynamic branch, of interest regarding the emergence of biological homochirality in origin of life research, and provides a relevant proof of concept for this thermodynamic evolution theorem. In our opinion, this result opens up a window for the design of models and simulations for the study of the dynamics of complex systems and their interactions with the environment [37].

\section{ACKNOWLEDGMENT}

The authors acknowledge the coordinated research grant, Grant No. CTQ2017-87864-C2-1(2)-P (MINECO), Spain.

\section{APPENDIX A: THE ENTROPY BALANCE FOR OPEN-FLOW REACTION SCHEMES}

We deduce the physical interpretation of the bilinear expression in Eq. (21), that is, the sum over products of reaction rates times affinities yields the entropy production $\sigma \geqslant 0$ plus the entropy exchange $\sigma_{e}$ (per unit volume). We demonstrate this for a general reaction scheme subject to input and output volumetric flow terms.

The chemical reactions for $r$ reactions involving $n$ chemical species can be summarized as follows:

$$
\alpha_{1 j} X_{1}+\cdots+\alpha_{n j} X_{n} \stackrel{k_{j}}{\rightarrow} \beta_{1 j} X_{1}+\cdots+\beta_{n j} X_{n}, \quad j=1, \ldots, r,
$$

where the $X_{i}, 1 \leqslant i \leqslant n$, are the $n$ chemical species, and each $k_{j}>0$ is the reaction rate constant for the $j$ th reaction. Forward and reverse reactions are treated separately, and the above list (A1) can also include the irreversible pseudoreactions, that is, the input and output volumetric flows to and from the reactor. There can be a such a flow for each one of the species, $u=1, \ldots, n$ :

$$
\begin{gathered}
\stackrel{k_{\mathrm{in}, u}\left[X_{u}\right]_{\mathrm{in}}}{\rightarrow} X_{u} \quad \text { (inflow), } \\
X_{u} \stackrel{k_{\text {out }, u}}{\rightarrow} \text { (outflow). }
\end{gathered}
$$

The effective rate constants $k_{\mathrm{in}, u}=q / V=k_{\text {out }, u}$, where $q=$ $l / s$ is volume $l$ of fluid entering and exiting the reactor of volume $V$ per second, and $\left[X_{u}\right]_{\text {in }}$ is the fixed concentration of species $u$ flowing into the reactor. These fluid flows maintain the system volume $V$ constant. Inflows and outflows need not be "matched"; that is, a given species $u$ can flow out of the reactor, without necessarily flowing into it: This means we would set $k_{\mathrm{in}, u}=0$ for this species. All species generally flow out with their instantaneous concentration as determined by the reactions taking place within the reactor.

We can group the full set of all the $r$ irreversible transformations (A1) into one subset of $p$ pairs that comprise all the reversible reactions:

$$
\begin{aligned}
& \alpha_{1 j} X_{1}+\cdots+\alpha_{n j} X_{n} \stackrel{k_{j}^{+}}{\rightarrow} \beta_{1 j} X_{1}+\cdots+\beta_{n j} X_{n}, \\
& \beta_{1 j} X_{1}+\cdots+\beta_{n j} X_{n} \stackrel{k_{j}^{-}}{\rightarrow} \alpha_{1 j} X_{1}+\cdots+\alpha_{n j} X_{n},
\end{aligned}
$$

for $j=1,2, \ldots, p$. Let $v_{i j}^{+}=\beta_{i j}-\alpha_{i j}, v_{i j}^{-}=-v_{i j}^{+}$be the elements of the stoichiometric matrix defined for $j$ in this range. The remaining transformations belong to the second subset and correspond to the input and output flow terms (A2) and (A3) (see below). 
The columns of the overall $n \times 2 p$ stoichiometic matrix corresponding this the set of $2 p$ (irreversible) transformations (A4) and (A5) are built from alternating (or dovetailing) the columns of the above submatrices $\boldsymbol{v}^{+}$and $\boldsymbol{v}^{-}$, as follows:

$$
v_{i l}=\left\{\begin{array}{lc}
v_{i,\left[\frac{l}{2}\right]+1}^{+} & l=1,3, \ldots, 2 p-1 \text { (odd), } \\
v_{i, \frac{l}{2}}^{-} & l=2,4, \ldots, 2 p(\text { even }) .
\end{array}\right.
$$

The remaining $r-2 p$ transformations in (A1) will represent the input-output flows; see below.

Forward, reverse rates of reaction and their corresponding affinities are $(j=1,2, \ldots, p)$

$$
\begin{gathered}
w_{j}^{+}=k_{j}^{+} \prod_{u=1}^{n}\left(x_{u}\right)^{\alpha_{u j}}, \\
w_{j}^{-}=k_{j}^{-} \prod_{u=1}^{n}\left(x_{u}\right)^{\beta_{u j}}, \\
A_{j}^{+}=-\sum_{k=1}^{n} v_{k j}^{+} \mu_{k}, \\
A_{j}^{-}=-\sum_{k=1}^{n} v_{k j}^{-} \mu_{k}=-A_{j}^{+} .
\end{gathered}
$$

Consider the following sum over the $p$ pairs of forward and reverse reactions:

$$
\begin{aligned}
\frac{1}{T} \sum_{k=1}^{2 p} w_{k} A_{k} & =\frac{1}{T} \sum_{j=1}^{p}\left(w_{j}^{+} A_{j}^{+}+w_{j}^{-} A_{j}^{-}\right) \\
& =\sum_{j=1}^{p}\left(w_{j}^{+}-w_{j}^{-}\right) \frac{A_{j}^{+}}{T} .
\end{aligned}
$$

Evaluate the affinities employing the relative chemical potentials (4):

$$
\begin{aligned}
\frac{A_{j}^{+}}{T} & =-\frac{1}{T} \sum_{k=1}^{n} v_{k j}^{+} \mu_{k}, \\
& =-R \sum_{k=1}^{n}\left(\beta_{k j}-\alpha_{k j}\right) \ln \left(\frac{\left[x_{k}\right]}{\left[x_{k}\right]^{\mathrm{eq}}}\right), \\
& =R \ln \left(\prod_{k=1}^{n}\left(\frac{\left[x_{k}\right]_{\mathrm{eq}}}{\left[x_{k}\right]}\right)^{\beta_{k j}-\alpha_{k j}}\right), \\
& =R \ln \left(\prod_{k=1}^{n}\left(\frac{\left[x_{k}\right]_{\mathrm{eq}}}{\left[x_{k}\right]}\right)^{\beta_{k j}}\left(\frac{\left[x_{k}\right]}{\left[x_{k}\right]_{\mathrm{eq}}}\right)^{\alpha_{k j}}\right), \\
& =R \ln \left(\prod_{k=1}^{n}\left(\frac{\left[x_{k}\right]_{\mathrm{eq}}^{\beta_{j k}}}{\left[x_{k}\right]_{\mathrm{eq}}^{\alpha_{j k}}}\right)^{n} \prod_{k=1}^{n}\left(\frac{\left[x_{k}\right]^{\alpha_{k j}}}{\left[x_{k}\right]^{\beta_{j k}}}\right)\right), \\
& =R \ln \left(\frac{k_{j}^{+}}{k_{j}^{-}} \prod_{k=1}^{n}\left(\frac{\left[x_{k}\right]^{\alpha_{k j}}}{\left[x_{k}\right]^{\beta_{j k}}}\right)\right), \\
& =R \ln \left(\frac{w_{j}^{+}}{w_{j}^{-}}\right) .
\end{aligned}
$$

Inserting (A18) into the final expression in (A11) shows that this sum (A11), over products of reaction rates for the reversible transformations times their corresponding affinities, gives the entropy production per unit volume $\sigma \geqslant 0$ [Eq. (2)] corresponding to the set of all the $p$-reversible reactions.

Next, consider the sum over the pseudoreactions. The columns of the overall $n \times 2 n$ stoichiometic matrix corresponding to the set of the $2 n$ (irreversible) pseudoreactions (A2) and (A3) are assembled from alternating (or dovetailing) the columns of the following sub-matrices, indicated as follows:

$$
v_{i l}^{\text {pseudo }}= \begin{cases}\delta_{i,\left[\frac{l}{2}\right]+1} & l=1,3, \ldots, 2 n-1 \text { (odd) }, \\ -\delta_{i, \frac{l}{2}} & l=2,4, \ldots, 2 n(\text { even }) .\end{cases}
$$

Define the reaction rates [the flows $J_{i}^{\text {flow }}$ in Eq. (21)] and affinities [the forces $X_{i}^{\text {flow }}$ in Eq. (21)] associated with the open flow terms:

$$
\begin{gathered}
w_{j}^{+}=k_{j}^{\text {in }}\left[X_{j}\right]_{\text {in }}, A_{j}^{\text {in }}=-\mu_{j}^{\text {rel }}, \\
w_{j}^{-}=k_{j}^{\text {out }}\left[X_{j}\right], \quad A_{j}^{\text {out }}=\mu_{j}^{\text {rel }} .
\end{gathered}
$$

Then, the part of the (overall) sum that runs over the pseudoreactions (A2) and (A3) can be written as follows (note the summation limits):

$$
\begin{gathered}
\frac{1}{T} \sum_{j=1}^{n}\left(w_{j}^{+} A_{j}^{\text {in }}+w_{j}^{-} A_{j}^{\text {out }}\right)=-\sum_{j=1}^{n}\left(w_{j}^{+}-w_{j}^{-}\right) \frac{\mu_{j}^{\text {rel }}}{T} \\
=-\sum_{j=1}^{n}\left(k_{j}^{\text {in }}\left[X_{j}\right]_{\text {in }}-k_{j}^{\text {out }}\left[X_{j}\right]\right) \frac{\mu_{j}^{\text {rel }}}{T}=\sigma_{e} .
\end{gathered}
$$

Together (A11), (A18), and (A23) demonstrate that products of reaction rates times affinities, summed over all the internal reactions and the volumetric flow terms, gives the sum of the entropy production and the entropy exchange (per unit volume):

$$
\sum_{k=1}^{r} w_{k} \frac{A_{k}}{T}=\sigma+\sigma_{e}
$$

\section{APPENDIX B: SYMMETRIES AND INVARIANCE OF $\Psi$}

We can express $\Psi$ in a manifestly symmetric way by defining a matrix with elements $G_{m n}=w_{n} v_{m n} / \sqrt{x_{m}}$. Then we have

$$
\begin{aligned}
\Psi & =-R \sum_{j=1}^{r} \sum_{l=1}^{r}\left(\sum_{k=1}^{n} \frac{w_{j} v_{k j} v_{k l} w_{l}}{x_{k}}\right), \\
& =-R \sum_{j=1}^{r} \sum_{l=1}^{r} \sum_{k=1}^{n} G_{j k}^{T} G_{k l}, \\
& =-R \sum_{j=1}^{r} \sum_{l=1}^{r}\left[G^{T} G\right]_{j l} \leqslant 0,
\end{aligned}
$$

where $M=\left[G^{T} G\right] \geqslant 0$ is a Gram matrix [21] and so is positive semidefinite. Thus, $\Psi \leqslant 0$. We transform the individual $G$ matrices by an orthogonal transformation (rotations) as 
follows:

$$
\begin{aligned}
G^{\prime} & =O G, \quad G^{T}=G^{T} O^{T}, \\
G^{T} G^{\prime} & =G^{T} O^{T} O G=G^{T} G, \\
\Rightarrow \Psi^{\prime} & =\Psi .
\end{aligned}
$$

Thus, $\Psi$ is invariant under rotations. We can express the inequality as a scalar product of a vector with itself, as follows [using Eq. (23)]:

$$
\Psi=-R \frac{d \boldsymbol{x}}{\sqrt{\boldsymbol{x}} d t} \cdot \frac{d \boldsymbol{x}}{\sqrt{\boldsymbol{x}} d t}=-R\left\|\frac{d \boldsymbol{x}}{\sqrt{\boldsymbol{x}} d t}\right\|^{2} \leqslant 0,
$$

where the components of the vector in question are defined by

$$
\left(\frac{d \boldsymbol{x}}{\sqrt{\boldsymbol{x}} d t}\right)_{k}=\sum_{l=1}^{r} \frac{v_{k l} w_{l}}{\sqrt{x_{k}}} .
$$

The expression for $\Psi$ (B5) is manifestly invariant under rotations. The $k$ th component of the vector is the rate of change of the concentration of the $k$ th species divided by the square root of its concentration (B6).

\section{APPENDIX C: OPEN-FLOW FRANK SCHEME}

The fully reversible Frank model scheme in an open-flow reactor of volume $V$, where $A$ flows in at constant concentration $[A]_{\text {in }}$ and all species $A, L, D$, and $P$ flow out with their corresponding instantaneous concentrations (see Fig. 1), is specified by the 15 transformations as numbered:

$$
\begin{aligned}
& A+L \underset{k-a(2)}{\stackrel{k_{a}(1)}{\rightleftharpoons}} L+L, \quad A+D \underset{k_{-a}(6)}{\stackrel{k_{a}(5)}{\rightleftharpoons}} D+D, \\
& A \underset{k_{-d}(4)}{\stackrel{k_{d}(3)}{\rightleftharpoons}} L, \quad A \underset{k_{-d}(8)}{\stackrel{k_{d}(7)}{\rightleftharpoons}} D \\
& L+D \underset{k_{-1}(10)}{\stackrel{k_{1}(9)}{\rightleftharpoons}} P, \\
& \bar{\emptyset} \stackrel{k_{f}[A]_{\text {in }}(11)}{\longrightarrow} A, \\
& A \stackrel{k_{f}(12)}{\longrightarrow} \emptyset, \\
& L \stackrel{k_{f}(13)}{\longrightarrow} \emptyset, \\
& D \stackrel{k_{f}(14)}{\longrightarrow} \emptyset, \\
& P \stackrel{k_{f}(15)}{\longrightarrow} \emptyset,
\end{aligned}
$$

where $k_{f}=q / V$, the volumetric flow rate $q=10^{-3} \mathrm{~L} / \mathrm{s}$, and $V=1 \mathrm{~L}$. For convenience, we number the 15 transformations as indicated for the purposes of carrying out the stoichiometric network analysis (SNA) [13] that follows below. Equality of the equilibrium constants for the direct production (C2) and the autocatalytic steps (C1) implies the constraint for the reaction rate constants:

$$
\frac{k_{a}}{k_{-a}}=\frac{k_{d}}{k_{-d}} .
$$

These transformations lead to the following set of differential rate equations for the concentrations and in the mean-field approximation:

$$
\begin{gathered}
\frac{d[A]}{d t}=-2 k_{d}[A]+k_{-d}([D]+[L])-k_{a}[A]([L]+[D]) \\
+k_{-a}\left([L]^{2}+[D]^{2}\right)+k_{f}\left([A]_{\text {in }}-[A]\right), \\
\frac{d[L]}{d t}=k_{d}[A]-k_{-d}[L]+k_{a}[A][L]-k_{-a}[L]^{2} \\
\quad-k_{1}[L][D]+k_{-1}[P]-k_{f}[L], \\
\frac{d[D]}{d t}= \\
\quad k_{d}[A]-k_{-d}[D]+k_{a}[A][D]-k_{-a}[D]^{2} \\
\quad-k_{1}[L][D]+k_{-1}[P]-k_{f}[D], \\
\frac{d[P]}{d t}=k_{1}[L][D]-k_{-1}[P]-k_{f}[P] .
\end{gathered}
$$

These imply the constraint

$$
\begin{aligned}
& \frac{d}{d t}([A]+[L]+[D]+2[P]) \\
& \quad=k_{f}\left([A]_{\text {in }}-[A]-[L]-[D]-2[P]\right),
\end{aligned}
$$

so that in the steady states, the total chemical mass in the reactor is equal to the input mass $[A]_{\text {in }}$ :

$$
[A]_{\text {in }}=[A]+[L]+[D]+2[P] .
$$

The differential equations $(\mathrm{C} 10)-(\mathrm{C} 13)$ are integrated numerically using the MATHEMATICA function NDSolve with options MaxSteps $\rightarrow 10^{6}$ and WorkingPrecision $\rightarrow 30$. All the initial conditions and parameters are specified with SetPrecision set to 30. The output InterpolatingFunctions is used to evaluate the dynamics, the enantiomeric excess, the entropy production, and the entropy exchange.

\section{Stoichiometric network analysis}

The associated stoichiometric matrix is [columns represent the transformations numbered consecutively from (1) to (15) in going from left to right, whereas the rows correspond to the four species $A, L, D$, and $P$ in labeling top to bottom]

$$
\boldsymbol{v}=\left(\begin{array}{ccccccccccccccc}
-1 & 1 & -1 & 1 & -1 & 1 & -1 & 1 & 0 & 0 & 1 & -1 & 0 & 0 & 0 \\
1 & -1 & 1 & -1 & 0 & 0 & 0 & 0 & -1 & 1 & 0 & 0 & -1 & 0 & 0 \\
0 & 0 & 0 & 0 & 1 & -1 & 1 & -1 & -1 & 1 & 0 & 0 & 0 & -1 & 0 \\
0 & 0 & 0 & 0 & 0 & 0 & 0 & 0 & 1 & -1 & 0 & 0 & 0 & 0 & -1
\end{array}\right)
$$

Then the intersection of the null space of the stoichiometric matrix $\boldsymbol{v}$ with the positive orthant of the 15-dimensional Euclidean space $R_{+}^{15}$ yields 18 currents $\boldsymbol{E}_{i}$ or the extreme flux modes (EFM) [13], which can be organized into the columns of the following 
matrix $\boldsymbol{E}$ (see Table II for the different reaction pathways they represent):

$$
\boldsymbol{E}=\left(\begin{array}{llllllllllllllllll}
0 & 0 & 0 & 0 & 0 & 0 & 1 & 0 & 1 & 0 & 0 & 0 & 0 & 1 & 0 & 0 & 1 & 1 \\
0 & 0 & 0 & 0 & 0 & 0 & 0 & 1 & 1 & 0 & 0 & 0 & 0 & 0 & 0 & 0 & 0 & 0 \\
0 & 0 & 0 & 0 & 0 & 1 & 0 & 1 & 0 & 0 & 0 & 0 & 1 & 0 & 1 & 1 & 0 & 0 \\
0 & 0 & 0 & 0 & 0 & 1 & 1 & 0 & 0 & 0 & 0 & 0 & 0 & 0 & 0 & 0 & 0 & 0 \\
0 & 0 & 1 & 0 & 1 & 0 & 0 & 0 & 0 & 0 & 0 & 1 & 0 & 0 & 0 & 1 & 0 & 1 \\
0 & 0 & 0 & 1 & 1 & 0 & 0 & 0 & 0 & 0 & 0 & 0 & 0 & 0 & 0 & 0 & 0 & 0 \\
0 & 1 & 0 & 1 & 0 & 0 & 0 & 0 & 0 & 0 & 1 & 0 & 0 & 0 & 1 & 0 & 1 & 0 \\
0 & 1 & 1 & 0 & 0 & 0 & 0 & 0 & 0 & 0 & 0 & 0 & 0 & 0 & 0 & 0 & 0 & 0 \\
1 & 0 & 0 & 0 & 0 & 0 & 0 & 0 & 0 & 0 & 0 & 0 & 0 & 0 & 1 & 1 & 1 & 1 \\
1 & 0 & 0 & 0 & 0 & 0 & 0 & 0 & 0 & 0 & 0 & 0 & 0 & 0 & 0 & 0 & 0 & 0 \\
0 & 0 & 0 & 0 & 0 & 0 & 0 & 0 & 0 & 1 & 1 & 1 & 1 & 1 & 2 & 2 & 2 & 2 \\
0 & 0 & 0 & 0 & 0 & 0 & 0 & 0 & 0 & 1 & 0 & 0 & 0 & 0 & 0 & 0 & 0 & 0 \\
0 & 0 & 0 & 0 & 0 & 0 & 0 & 0 & 0 & 0 & 0 & 0 & 1 & 1 & 0 & 0 & 0 & 0 \\
0 & 0 & 0 & 0 & 0 & 0 & 0 & 0 & 0 & 0 & 1 & 1 & 0 & 0 & 0 & 0 & 0 & 0 \\
0 & 0 & 0 & 0 & 0 & 0 & 0 & 0 & 0 & 0 & 0 & 0 & 0 & 0 & 1 & 1 & 1 & 1
\end{array}\right),
$$

and obeys the matrix product $\boldsymbol{v E}=\mathbf{0}$.

[1] P. Glansdorff and I. Prigogine, Thermodynamic Theory of Structure, Stability, and Fluctuations (Wiley, New York, 1971).

[2] G. Nicolis and I. Prigogine, Self-Organization in Nonequilibrium Systems (Wiley, New York, 1977).

[3] D. Kondepudi and I. Prigogine, Modern Thermodynamics, 2nd ed. (Wiley, New York, 2015).

[4] A. Kleidon and R. D. Lorenz, editors, Non-equilibrium Thermodynamics and the Production of Entropy: Life, Earth, and Beyond (Springer, Heidelberg, 2005).

[5] A. Bejan, Maxwell's demons everywhere: Evolving design as the arrow of time, Sci. Rep. 4, 4017 (2014).

[6] Z. Yoshida and S. M. Mahajan, "Maximum" entropy production in self-organized plasma boundary layer: A thermodynamic discussion about turbulent heat transport, Phys. Plasmas 15, 032307 (2008).

[7] R. D. Lorenz, J. I. Lunine, P. G. Withers, and C. P. McKay, Titan, Mars, and Earth: Entropy production by latitudinal heat transport, Geophys. Res. Lett. 28, 415 (2001).

[8] Y. Yang, S. Bruns, S. L. S. Stipp, and H. O. Sørensen, Patterns of entropy production in dissolving natural porous media with flowing fluid, PLoS ONE 13, e0204165 (2018).

[9] M. del Jesus, R. Foti, A. Rinaldo, and I. Rodriguez-Iturbe, Maximum entropy production, carbon assimilation, and the spatial organization of vegetation in river basins, Proc. Natl. Acad. Sci. USA 109, 20837 (2012).

[10] A. I. Zotin and R. S. Zotina, Thermodynamic aspects of developmental biology, J. Theor. Bio. 17, 57 (1967).

[11] D. E. Rosner, Transport Processes in Chemically Reacting Flow Systems (Butterworths, Boston, 1986).

[12] A. Blokhuis, D. Lacoste, and P. Gaspard, Reaction kinetics in open reactors and serial transfers between closed reactors, J. Chem. Phys. 148, 144902 (2018).

[13] B. L. Clarke, Stoichiometric network analysis, Cell Biophys. 12, 237 (1988).

[14] D. Hochberg, R. D. Bourdon García, J. A. Ágreda Bastidas, and J. M. Ribó, Stoichiometric network analysis of spontaneous mirror symmetry breaking in chemical reactions, Phys. Chem. Chem. Phys. 19, 17618 (2017).
[15] F. C. Frank, On spontaneous asymmetric synthesis, Biochim. Biophys. Acta 11, 459 (1953).

[16] D. Hochberg and J. M. Ribó, Stoichiometric network analysis of entropy production in chemical reactions, Phys. Chem. Chem. Phys. 20, 23726 (2018).

[17] H. Mahara and T. Yamaguchi, Entropy balance in distributed reversible Gray-Scott model, Phys. D (Amsterdam, Neth.) 239, 729 (2010).

[18] L. Onsager, Reciprocal relations in irreversible processes. I., Phys. Rev. 37, 405 (1931).

[19] P. Glansdorff and I. Prigogine, Sur les propriétés différentielles de la production d'entropie, Physica 20, 773 (1954); On a general evolution criterion in macroscopic physics, 30, 351 (1964).

[20] A. C. Sicardi Schifino, A. Costa, and C. Ferro Fontán, Generalization of the Glansdorff-Prigogine stability criterion, J. Math. Phys. 32, 1350 (1991).

[21] H. Schwerdtfeger, Introduction to Linear Algebra and the Theory of Matrices (P. Noordhoff, Groningen, 1950).

[22] An inequality allowing for time-dependent external mechanical forces has been derived [19], while still maintaining the assumption of vanishing boundary conditions.

[23] D. Hochberg and J. M. Ribó, Entropic analysis of mirror symmetry breaking in chiral hypercycles, Life 9, 28 (2019).

[24] K. Soai, T. Kawasaki, and A. Matsumoto, Asymmetric autocatalysis of pyrimidyl alkanol and its application to the study on the origin of homochirality, Acc. Chem. Res. 47, 3643 (2014).

[25] D. Kondepudi and L. Kapcha, Entropy production in chiral symmetry breaking transitions, Chirality 20, 524 (2008).

[26] M. Mauksch and S. B. Tsogoeva, Spontaneous emergence of homochirality via coherently coupled antagonistic and reversible reaction cycles, ChemPhysChem 9, 2359 (2008).

[27] R. Plasson and H. Bersini, Energetic and entropic analysis of mirror symmetry breaking processes in a recycled microreversible chemical system, J. Phys. Chem. B 113, 3477 (2009).

[28] C. Blanco and D. Hochberg, Chiral polymerization: Symmetry breaking and entropy production in closed systems, Phys. Chem. Chem. Phys. 13, 839 (2011).

[29] C. Blanco, J. M. Ribó, J. Crusats, Z. El-Hachemi, A. 
Moyano, and D. Hochberg, Mirror symmetry breaking with limited enantioselective autocatalysis and temperature gradients: A stability survey, Phys. Chem. Chem. Phys. 15, 1546 (2013).

[30] J. M. Ribó and D. Hochberg, Chemical basis of biological homochirality during the abiotic evolution stages on earth, Symmetry 11, 814 (2019).

[31] D. Kondepudi and Z. Mundy, Spontaneous chiral symmetry breaking and entropy production in a closed system, Symmetry 12, 769 (2020).

[32] J. Schnakenberg, Network theory of microscopic and macroscopic behavior of master equation systems, Rev. Mod. Phys. 48, 571 (1976).

[33] L. Jiu-li, C. Van den Broeck, and G. Nicolis, Stability criteria and fluctuations around nonequilibrium states, Z. Phys. B 56, 165 (1984).

[34] C. Y. Mou, J.-L. Luo, and G. Nicolis, Stochastic thermodynamics of nonequilibrium steady states in chemical reaction systems, J. Chem. Phys. 84, 7011 (1986).

[35] P. Gaspard, Fluctuation theorem for nonequilibrium reactions, J. Chem. Phys. 120, 8898 (2004).

[36] J. M. Ribó and D. Hochberg, Spontaneous mirror symmetry breaking: An entropy production survey of the racemate instability and the emergence of stable scalemic stationary states, Phys. Chem. Chem. Phys. 22, 14013 (2020).

[37] P. Gaspard and R. Kapral, Nonequilibrium thermodynamics and boundary conditions for reaction and transport in heterogeneous media, J. Chem. Phys. 148, 194114 (2018). 